\title{
Metodología para el diseño participativo de un recurso basado en TIC, como herramienta de desarrollo de un proyecto con impacto rural
}

\author{
Diana Duarte ${ }^{1}$, Mauricio Peralta², Breghtness Vera ${ }^{3}$, José Cortés ${ }^{4}$, Manuel Herrera ${ }^{5}$
}

Recibido: Agosto 29 de 2014 Aprobado: Diciembre 11 de 2014

\begin{abstract}
Resumen:
Aunque las Tecnologías de la Información y las Comunicaciones (TIC) se configuran en la actualidad como estratégicas para el fomento efectivo de iniciativas cuyos objetivos se alinean con la búsqueda del desarrollo rural, es necesario señalar que las TIC no se pueden considerar como la solución sino como una herramienta de apoyo para dichas iniciativas. Teniendo en cuenta lo anterior, la adecuada apropiación y efectividad de un recurso basado en TIC requiere que su diseño e implementación comprenda no solo los requerimientos funcionales de la tecnología sino el contexto y las razones de fondo para su creación. El presente artículo expone la metodología para el diseño e implementación de una página WEB dentro del contexto del proyecto "Fortalecimiento de Negocios Verdes, Provincia del Guavio (Cundinamarca)" iniciativa que tiene como objetivo principal generar una red de integración sostenible en los municipios de Guasca, Gachetá y Junín. La página WEB busca transformarse en una plataforma de intercambio comercial que a futuro contará con las funcionalidades necesarias para potencializar la región del Guavio como el principal polo de Negocios Verdes de Colombia. El artículo presenta como resultado algunas estrategias que se han generado a partir de la aplicación de la metodología, que reflejan cómo gracias a esta, el diseño de la página WEB ha requerido adelantar gestiones que van mucho más allá del diseño tecnológico.
\end{abstract}

Palabras clave: Tecnologías de la información y las comunicaciones, Páginas WEB, Desarrollo rural, Negocios verdes.

\begin{abstract}
:
Although the Information and Communication Technologies (ICT) nowadays are considered as strategic tools in the effective achievement of projects that look for the rural development, it is necessary to point out that the ICT are not the solution by itself. According with the previous, the ICT design and its implementation has to include not only the technical requirements, but also it has to be developed taking into account the contexts where it'll be used. This paper presents the ICT design methodology that was used by the project "Strengthening of Green Business at the Guavio Region" which goal is the generation of a sustainable network in the towns of Guasca, Gacheta and Junin. The results show a list of strategies that have been generated after its implementation that allows the suitable usage of the ICT.
\end{abstract}

Keywords: Information and Communication Technology, WEB pages, Rural development, Green Businees.

\footnotetext{
${ }^{1}$ Ingeniera Industrial, máster en Ingeniería Industrial, docente investigadora de UNIMINUTO, Grupo de Investigación en Desarrollo Regional.

2 Especialista en Manejo Integrado del Medio Ambiente, máster en Ingeniería y Gerencia en Construcción, UNIMINUTO, Grupo de Investigación en Desarrollo Regional.

${ }^{3}$ Ingeniero en Agroecología, investigador Junior UNIMINUTO, Grupo de Investigación en Desarrollo Regional.

${ }^{4}$ Trabajador Social, investigador Auxiliar UNIMINUTO, Grupo de Investigación en Desarrollo Regional.

${ }^{5}$ Estudiante Ingeniería Agroecológica, investigador Auxiliar UNIMINUTO, Grupo de Investigación en Desarrollo Regional.
} 


\section{INTRODUCCIÓN}

Dentro los planes nacionales del gobierno actual prevalece la idea del desarrollo rural. Las TIC o Tecnologías de la Información y las Comunicaciones tienen un rol fundamental en este sentido, al contribuir con la modernización y revitalización de las actividades productivas tradicionales de las regiones. Las nuevas plataformas y redes de información, como las páginas Web, están transformando de manera significativa los sistemas productivos y la toma de decisiones en sectores como la minería, la pesca, la agricultura y el turismo rural (Naser \& Concha, 2014). Sin embargo, para que su uso en procesos que propenden por el desarrollo en zonas rurales sea efectivo, se debe tener en cuenta en su diseño e implementación el contexto y las características funcionales necesarias para su correcta apropiación. Partiendo de lo anterior, en el proyecto Fortalecimiento de Negocios Verdes Comunitarios en El Guavio (Cundinamarca) se diseñó y desarrolló una página WEB, cuya metodología se estableció no simplemente a partir del cumplimiento de los requerimientos funcionales de un pequeño grupo de actores, sino buscando satisfacer los intereses y expectativas de los diferentes grupos de involucrados: instituciones formuladoras y financiadoras y beneficiarios. Es así como el producto es un entrecruzamiento de perspectivas que se fueron incorporando progresivamente por medio de ciclos de realimentación anclados a procesos de validación, verificación y modelación.

\section{TECNOLOGÍAS DE LA INFORMACIÓN Y DE LAS COMUNICACIONES}

Según el artículo $6^{\circ}$ de la Ley 1341 de 2009, para el Ministerio de las Tecnologías de la Información y las Comunicaciones (MinTIC), las TIC se definen como las herramientas, equipos, aplicaciones o redes cuya utilización permite el procesamiento, almacenamiento y transmisión de información (texto, voz, datos e imágenes principalmente). Enfatizando lo anterior, las TIC están íntimamente ligadas a la utilización de medios básicos como la informática, la microelectrónica y las telecomunicaciones, que tienen como fin almacenar, procesar y difundir todo tipo de información (Parlamento Andino, 2012).

El sector de las TIC se ha desarrollado de forma acelerada en los últimos 20 años; en la actualidad el sector incluye software, hardware, telefonía, la Internet, contenidos, aplicaciones y servicios de soporte cuyos proveedores pueden ser desde compañías transnacionales hasta pequeños emprendedores (Kramer \& Jenkins, 2007).
En Colombia, aunque las TIC son incluidas por primera vez dentro del Plan Nacional de Desarrollo en el periodo presidencial 1998-2002 (Departamento Nacional Planeación, 1998) el principal ente que promueve su uso y apropiación es el MinTIC. Un ejemplo claro de su accionar es el plan "Vive Digital", el cual busca que Colombia sea reconocida a nivel mundial por su liderazgo en el desarrollo de aplicaciones orientadas hacia la resolución de problemáticas o necesidades de grupos en situación de vulnerabilidad (Ministerio de Educación Nacional, 2014).

\section{LAS TIC Y EL DESARROLLO RURAL}

En líneas generales, una iniciativa que esté orientada a la generación de desarrollo rural debe enmarcar sus actividades dentro de objetivos tales como: mejorar la competitividad del sector, asegurar la administración sostenible de recursos naturales y definir estrategias de balance territorial entre el desarrollo de las economías y la calidad de vida de las comunidades que lo habitan (European Commission, 2014).

Las TIC se convierten en herramientas estratégicas para la consecución de objetivos como los ya mencionados, al tener la potencialidad de modernizar y revitalizar las actividades productivas tradicionales de las regiones. Las TIC permiten el mejoramiento y seguimiento de los procesos de cosecha y comercialización, la medición de riesgos asociados al clima, a los precios, y facilitan transacciones para el desarrollo de innovaciones a lo largo de una red o cadena productiva (CEPAL, FAO \& IICA, 2011).

Sin embargo, es conveniente mencionar que las TIC no son en sí mismas la solución a los problemas; son herramientas que facilitan su resolución. Es debido a lo anterior que cuando se aspira a tener un uso efectivo de las TIC como operadores de desarrollo rural, es necesario que en su diseño y proceso de apropiación se tenga en cuenta tanto el contexto social, económico y organizativo donde se realiza, como la estructura de operación. (Bossio, López Velarde, Saravia, \& Wolf, 2005).

En cuanto el contexto es importante considerar un factor de relevancia para el uso efectivo de las TIC, cual es el nivel de interconexión. Para el caso de Cundinamarca, según el MinTic, el Departamento se ubicó en el puesto 12 entre los 33 nodos principales (32 departamentos y Bogotá D.C.) con un 6,22\% de penetración en Internet, a diferencia de Bogotá D.C. que se ubicó en el primer lugar con un $16,45 \%$. Teniendo en cuenta realidades como la anterior, 
cuando las TIC son diseñadas como instrumentos de inclusión y desde la consideración del contexto de las necesidades locales, su uso (Antolín Larios, 2009):

- Dinamiza las relaciones comerciales.

- Estrecha o restablece vínculos familiares al facilitar la comunicación con los migrantes.

- Reduce la sensación de abandono producida por falta de comunicación.

- Promueve el acercamiento con los planificadores gubernamentales y los organismos de desarrollo.

- Fomenta la participación de las comunidades en las decisiones que afectan sus vidas.

- Contribuye a coordinar esfuerzos de desarrollo local, regional y nacional.

- Contribuye a la generación de conocimiento.

Este último aspecto se puede considerar como una perspectiva en la que se basa el desarrollo rural a partir de la generación y gestión de conocimiento. Para la efectividad de tales procesos es necesario, en primera instancia, identificar aquel conocimiento que no es objetivo, sino que surge de interpretaciones y percepciones individuales dentro de una organización; en segunda instancia permitir que la información se encuentre disponible para su uso (Nonaka, 1995). Para llevar a cabo el anterior mecanismo, el Estado cobra un rol importante al tener la capacidad de crear los incentivos necesarios para que personas e instituciones fomenten manifestaciones tanto culturales como científicas, que al apoyarse en las TIC, logran amplificar su capacidad y su gestión (Naser \& Concha, 2014).

A partir de lo anterior se puede inferir que las TIC como herramientas para la gestión de iniciativas que buscan el desarrollo rural por medio del conocimiento, deben estar estructuradas de forma que permitan administrar la complejidad que se genera a partir de la interacción de múltiples actores y objetivos.

A continuación se presenta la metodología del proyecto Fortalecimiento de Negocios Verdes Provincia de El Guavio (Cundinamarca), la cual ha permitido diseñar una página WEB como paso inicial en la estructuración de una plataforma que a futuro, hará posible la gestión efectiva de los procesos de desarrollo rural que ya se adelantan en los municipios de Guasca, Gachetá y Junín.

\section{PROYECTO DE FORTALECIMIENTO DE NEGOCIOS VERDES COMUNITARIOS PROVINCIA DE EL GUAVIO}

La iniciativa tiene como objetivo fortalecer capacidades de innovación y emprendimiento en las comunidades de la Región de El Guavio, consolidando una red de integración sostenible, con oportunidades de generación de ingresos por medio de los denominados Negocios Verdes. El proyecto fue formulado en el año 2012 por el grupo de Ingenieros Sin Fronteras Colombia, constituido por la Universidad de los Andes y la Corporación Universitaria Minuto de Dios, y es ejecutado en el año 2014 mediante el Convenio 019 referido al Fortalecimiento de la Innovación a través del Parque Científico de Innovación Social UNIMINUTO y la Gobernación de Cundinamarca, financiado por el Fondo de Ciencia, Tecnología e Innovación del Sistema General de Regalías - SGR.

Como beneficiarios, el proyecto cuenta con la participación de 350 estudiantes de décimo y undécimo grado de secundaria de los municipios de Guasca, Gachetá y Junín, 35 unidades productivas de los mismos municipios y estudiantes universitarios de las instituciones de educación superior participantes. El proyecto se encuentra estructurado en cuatro líneas de trabajo, a) Fortalecimiento como Negocio Verde, b) Programa de Innovación-Acción desde los colegios, c) Proyecto investigación y publicaciones y, d) Desarrollo participativo de soluciones tecnológicas.

\subsection{Las TIC en el marco del proyecto}

Dentro de la última línea de trabajo enunciada en la sección anterior se enmarca la base para el logro del objetivo principal (consolidar una red de integración sostenible). La red, en el marco del desarrollo rural ya definido, se configura como una apuesta de organización multisectorial que permitirá aumentar el turismo y la afluencia de clientes hacia los municipios, por medio de la muestra integrada e innovadora de siete propuestas de valor que incluyen rutas turísticas, fincas agro-turísticas de aprendizaje, dos productos insignia: la quínoa y la miel, una aplicación móvil y la inclusión de los colegios y las universidades por medio de programas de práctica social y empresarial. Así mismo, se busca visibilizar a las unidades productivas de forma individual, como propuestas económicas que están alineando sus prácticas hacia la sostenibilidad.

Se desea anclar la red en una plataforma virtual que conecte los actores ya mencionados, de forma 
que se facilite la consolidación de un mercado con tales características en El Guavio. Sin embargo, para llegar a tal visión, se deben cumplir varias etapas que permitan la apropiación y adaptación progresiva por parte de los actores de la plataforma virtual. En tal sentido, la investigación de cómo se debe llevar a cabo lo anterior se debe construir con los mismos actores, es decir, la respuesta se debe dar de forma participativa, no impositiva.

Siendo así, el esquema que se ha construido a lo largo de la experiencia para la instauración de la plataforma ha sido el siguiente:

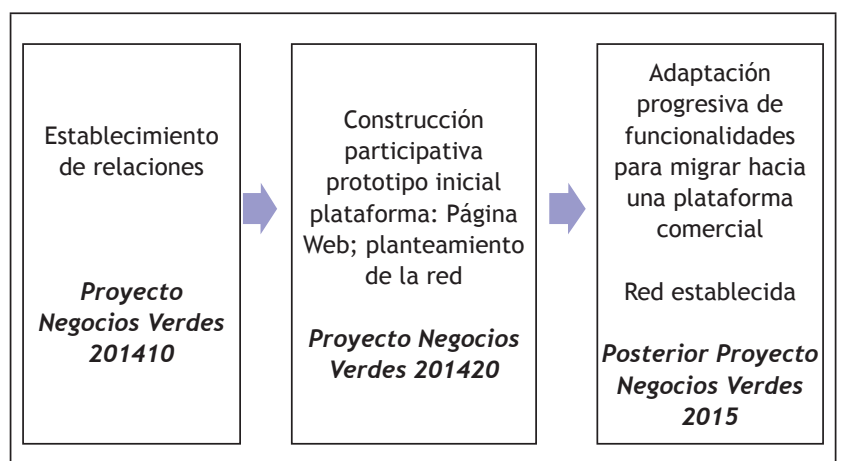

Figura 1. Etapas para el establecimiento de la plataforma. Fuente: los autores

Respecto a las dos primeras etapas, estas se estructuraron por medio de un proceso de construcción de relaciones y de participación que inició en el año 2013, y que concluyó en un evento denominado Laboratorio de Soluciones Verdes. Este evento permitió que los participantes, por medio de una elección democrática, pudieran seleccionar aquellas propuestas que representaban el interés colectivo y que ahora se configuran como parte de la apuesta de valor de la red.

La segunda etapa finaliza con lo que se denomina "Espacios de diseño participativo", donde cada una de las siete propuestas serán llevadas a prototipos prácticos que permitan dimensionarlas de forma rápida y por medio de la acción.

La complejidad de la ejecución de los espacios de diseño participativo subyace principalmente en que serán siete procesos que se llevarán de forma simultánea, en un periodo de un mes en los tres municipios (zonas geográficas diferentes) y que serán guiados por siete equipos conformados cada uno por cuatro participantes de diferentes perfiles; a lo anterior se suma la complejidad de los beneficiarios y sus intereses. Es a partir de dicha experiencia que la página WEB (futura plataforma) empieza a cobrar sentido para todos los actores, puesto que existe la evidente necesidad de llevar a cabo una adecuada gestión de la comunicación.

Para términos del presente artículo, se presenta a continuación la metodología que ha sido usada en el proyecto en la etapa dos, la cual ha permitido encontrar progresivamente, estrategias que serán necesarias llevar a cabo para una correcta apropiación y validez de la futura plataforma dentro del contexto establecido.

\subsection{Metodología}

La metodología que ha sido establecida para llevar a cabo todo el proceso de diseño e inclusión de la página Web con los requerimientos ya señalados, se muestra a continuación:

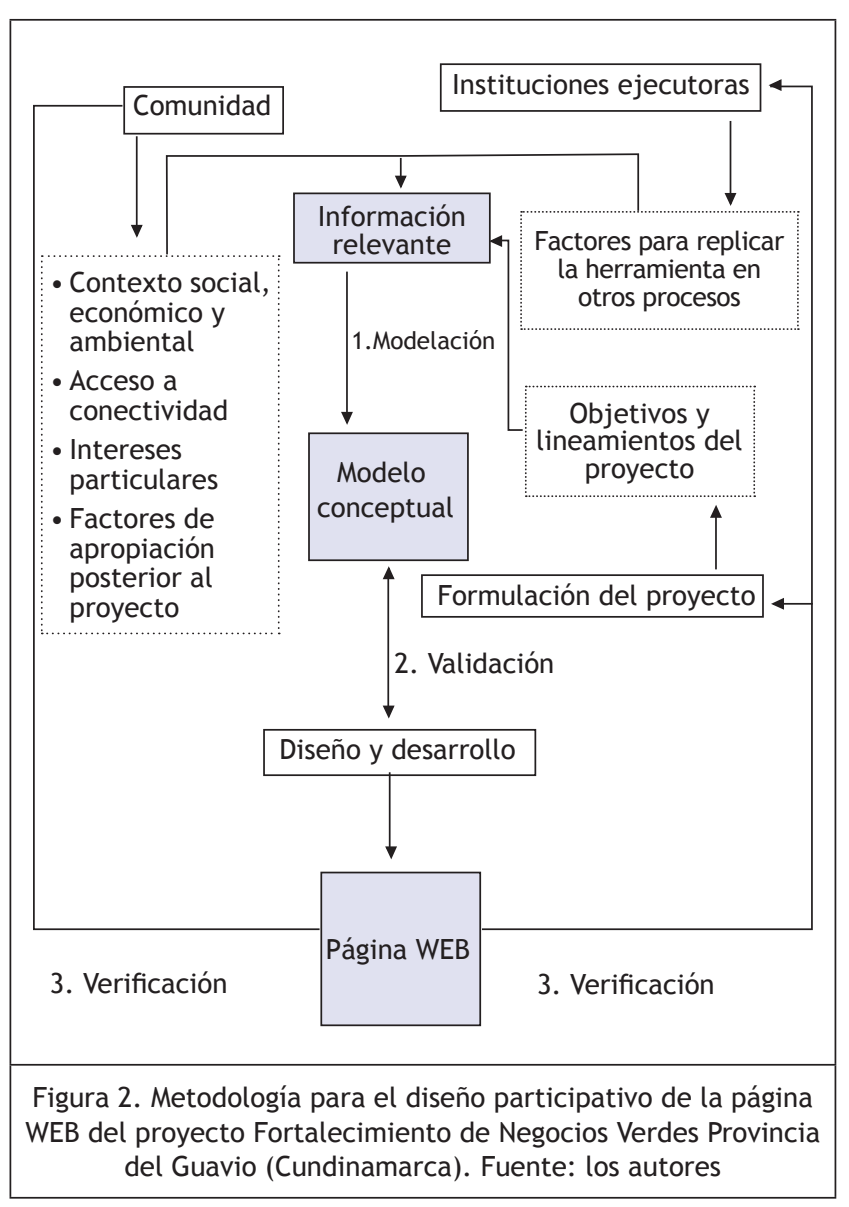

El modelo conceptual o representación de la página WEB surge a partir del análisis de la siguiente información relevante: los lineamientos del proyecto, los factores que permitan que sea replicada para otros procesos y las características de la comunidad que construye un modelo conceptual. Posterior al establecimiento del modelo inicial, se ingresa a los procesos de diseño y desarrollo de la página WEB, que se encuentran en constante validación. 
Así mismo y de forma paralela se hace la verificación respecto a los tres polos generadores de información relevante: la comunidad, las instituciones participantes y la formulación del proyecto. La verificación se hace tanto en contenido como en forma.

La metodología es iterativa y se realiza de forma paralela a su implementación, de forma que se garantice que los cambios estén siendo tenidos en cuenta en el desarrollo, a partir de la misma experiencia y no desde supuestos ajenos a la realidad.

\subsection{Resultados parciales de la metodología}

El proceso de definición de la información relevante se llevó a cabo a partir de la ejecución del mismo proyecto, es decir, fue un proceso iterativo que se basó en la experiencia de más de un año con los participantes. La página WEB ha estado en proceso de implementación y verificación simultánea desde la segunda semana de septiembre del 2014. Dicho proceso de verificación se ha realizado por medio de una estructura de ciclos de realimentación que han permitido que se hagan los ajustes necesarios para que la página WEB pueda estar, progresivamente, acorde con los intereses de los tres polos generadores de información relevante.

Así mismo, gracias a la aplicación de la metodología ha sido posible establecer las siguientes estrategias como necesarias para la correcta adaptación y apropiación de la página WEB, estrategias que deberán ser migradas también a la plataforma:

1. Partiendo del hecho de que en los tres municipios no hay cobertura total de Internet, ha sido necesario establecer rutinas para la socialización del contenido de la página WEB con los actores municipales.

2. Necesidad de realizar actividades de homologación de la página WEB con algunos procesos de la Plataforma Tecnológica del Parque Científico de Innovación Social, que se encontraba en diseño.

3. Necesidad de incluir un perfil individual para cada unidad productiva o participante, de forma que pueda visibilizar sus productos o servicios.

4. Diseño del concepto de la página WEB como una multimedia que represente los sectores ambiental, agrícola y rural.

También es necesario evidenciar resultados en términos de la gestión del conocimiento; para esto es preciso indicar que la información, antes de ser incorporada en la página WEB debió recorrer un proceso que parte de una comunicación oral en campo (un taller, una salida de campo, una reunión), que es plasmada en forma de acta o de reporte, para transformarse posteriormente en información publicable y finalmente ser introducida en la página WEB. Todo el proceso de gestión de conocimiento indicado requiere de un sistema de control debidamente estructurado, que propenda por generar espacios de comunicación, constantemente.

Lo anterior se puede considerar que ha sido el mayor cuello de botella en cuanto a la efectividad de la metodología, debido a que la cultura de gestión del conocimiento se ve truncada por los múltiples procesos paralelos llevados a cabo en el contexto del proceso, en donde ésta gestión se pone en segundo plano generando estancamientos de varios procesos de realimentación.

\section{CONCLUSIONES}

Las TIC se configuran como herramientas fundamentales para la gestión y el apoyo de procesos en múltiples dimensiones. Para el caso del desarrollo rural, las TIC pueden ser utilizadas como plataformas tanto para la optimización de procesos en el sector rural, como para el desarrollo de procesos de generación y divulgación de conocimiento que tengan como objetivo fortalecer las actividades comerciales en distintas zonas geográficas. Esto se evidenció en el caso del proyecto Fortalecimiento de Negocios Verdes Provincia de El Guavio (Cundinamarca), que encuentra desde el análisis y la experiencia que brinda la misma iniciativa, la necesidad inminente de apropiar una tecnología de información y comunicación para administrar la complejidad que genera en un periodo corto de tiempo, con la participación de actores de diversos perfiles, al igual que desarrollar de forma simultánea siete procesos que permitirán diseñar prototipos básicos de las propuestas que sustentan la consecución de su objetivo principal: generar una red de integración sostenible.

Alineándose con los conceptos presentados, la metodología usada en el proyecto parte de la selección de la información relevante en tres focos de interés para la página WEB: las instituciones ejecutoras, la comunidad y la formulación del proyecto, para entrar a partir de allí en un proceso de validación y verificación el cual inicia con un modelo conceptual y finaliza con la página Web. 
Para lograr efectividad, la metodología debe estar vinculada con un proceso ordenado y controlado de gestión del conocimiento, que establezca desde el principio y como prioridad, la documentación de la información.

En el desarrollo adecuado de un recurso basado en TIC, que busque ser apoyo en el desarrollo de proyectos que tienen como objetivo generar impactos en comunidades rurales, es preciso determinar inicialmente los grupos de actores y sus intereses a fin de contar con un modelo conceptual; por otra parte, tener en cuenta los procesos de realimentación de validación y verificación. Sin embargo, como ya se ha indicado, el desarrollo de estos procesos de realimentación debe estar acompañado de una efectiva gestión del conocimiento que garantice el flujo eficaz de la información: en este sentido se debe evitar la generación cuellos de botella que se traducen en retrasos en la ejecución de la metodología.

\section{REFERENCIAS BIBLIOGRÁFICAS}

1. Ministerio de Educación Nacional [MinEducación]. (9 de 8 de 2014). Ministerio de Educación Nacional. Recuperado el 9 de Agosto de 2014, de http://www.mineducacion.gov.co/1621/w3propertyvalue-39471.html

2. Antolín Larios, J. C. (2009). Inclusión o exclusión social: El reto de las TIC y el caso de las poblaciones rurales centroamericanas. Apuntes al rededor de la experiencia. Revista Científica de la Fundación iberoamericana para la excelencia educativa, 51.

3. Bossio, J. F., López Velarde, J., Saravia, M., \& Wolf, P. (2005). Desarrollo rural y tecnologías de información y comunicación. Experiencias en el Perú: Lecciones aprendidas y recomendaciones. Lima: Stampa Gráfica S.A.C.

4. CEPAL, FAO \& IICA. (2011). Perspectivas de la agricultura y del desarrollo rural en las Américas: Una mirada hacia América Latina $Y$ el Caribe. San José Costa Rica: Imprenta IICA, sede central.

5. Clarke, K., \& Primo, D. (2012). A Model Discipline: Political Science and the Logic of
Representations. Oxford: Oxford Scholarship Online.

6. Departamento Nacional Planeación. (1998). Página del Departamento Nacional Planeación. Obtenido de Plan Nacional de Desarrollo: https: / / www.dnp.gov.co/Plan-Nacional-deDesarrollo/Paginas/Planes-de-Desarrolloanteriores.aspx.

7. European Commission. (3 de 08 de 2014). Agriculture and rural development. Obtenido de http://ec.europa.eu/agriculture/rural-deve lopment-2014-2020/index_en.htm.

8. Goldstein, J., \& Hazy, J. (2010). Complexity and the Nexus of Leadership. New York: PALGRAVE MACMILLAN.

9. Kramer, W., \& Jenkins, B. (2007). The Role of the Information and Communications Technology Sector in Expanding Economic Opportunity. Cambridge: John F. Kennedy School of Goverment, Harvard University.

10. Ministerio de Tecnologías de la Información y las Comunicaciones [MinTic]. (20 de 08 de 2014). Glosario Ministerio de Tecnologías de la Información y las Comunicaciones. Obtenido de http://www.mintic.gov.co/portal/604/w3propertyvalue-1051.html\#ui-accordion-tabsheader-18

11. Naser, A., \& Concha, G. (2014). Rol de las TIC en la gestión pública y en la planificación para un desarrollo sostenible en América Latina y el Caribe. Santiago de Chile: Naciones Unidas CEPAL.

12. Nonaka, I. (1995). The knowledge-Creating Company. Oxford: Oxford University Press USA.

13. Parlamento Andino. (2012). Informe Ejecutivo "Tecnologías de la información y la comunicación (TICS). Publicaciones Parlamento Andino.

14. Reyes, A. (2001). ICTs and Modeling: Some Current Challenges from a Constructivist Approach. Lincoln: University of Lincoln. 